\title{
Development of measurable criteria to identify and prioritize patients for inclusion in comprehensive medication management programs within primary care settings
}

\author{
Martin A Bishop, PharmD, MS; Hsien-Yen Chang, PhD; Christopher Kitchen, MA, MS; Jonathan P Weiner, DrPH; \\ Hadi Kharrazi, MD, PhD; and Kenneth M Shermock, PharmD, PhD
}

\author{
What is already known \\ about this subject \\ - Medication use is nearly universal, \\ and pharmacists can improve the \\ safety and efficacy of medication use \\ through comprehensive medication \\ management (CMM). \\ - Identifying and prioritizing patients \\ for pharmacist review in a large \\ population in primary care or \\ population-based settings is typically \\ inefficient, relying on overly broad \\ criteria available in the patient \\ medical record, manual review, or \\ referral from other providers.
}

\author{
What this study adds \\ - Data from insurance claims can be \\ used to populate novel markers of \\ medication risk based on a patient's \\ medication use. \\ - Compared with the overall population \\ and with individuals meeting \\ medication therapy management \\ eligibility criteria, patients identified by \\ the novel markers had increased costs, \\ health care utilization, and gaps in \\ medication adherence. \\ - The novel medication risk markers can \\ be used to efficiently identify patients \\ with specific characteristics related to \\ medication use who could benefit from \\ pharmacist intervention to reduce costs \\ and/or acute care utilization.
}

\begin{abstract}
Author affiliations
Martin A Bishop, PharmD, MS, Department of Pharmacy, The Johns Hopkins Hospital, Baltimore, MD. Hsien-Yen Chang, PhD, Center for Population Health Information Technology, Center for Drug Safety and Effectiveness, Department of Health Policy and Management, The Johns Hopkins Bloomberg School of Public Health, Baltimore, MD. Christopher Kitchen, MA, MS; Jonathan P Weiner, DrPH; and Hadi Kharrazi, MD, PhD, Center for Population Health Information Technology, Department of Health Policy and Management, The Johns Hopkins Bloomberg School of Public Health, Baltimore, MD. Kenneth M Shermock, PharmD, PhD, Department of Pharmacy, The Johns Hopkins Health System, Baltimore, MD.
\end{abstract}

AUTHOR CORRESPONDENCE:

Martin A Bishop, 443.287.2395;

mbishop@jhmi.edu

J Manag Care Spec Pharm 2021;27(8):1009-18

Copyright $\odot 2021$, Academy of Managed Care Pharmacy. All rights reserved.

or automated mechanisms for identifying patients who would likely benefit from a pharmacist consultation. Existing tools to prioritize patients for pharmacist review often require manual chart review by a pharmacist or other clinicians or data collection by patient survey. 
OBJECTIVES: To (1) create new medication risk markers for identifying and prioritizing patients within a population and (2) identify patients who met these new markers, assess their clinical characteristics, and compare them with criteria that are widely used for medication therapy management (MTM).

METHODS: Along with published literature, a panel of subject matter experts informed the development of 3 medication risk markers. To assess the prevalence of markers developed, we used Multum, a medication database, for medication-level characteristics, and for patient-level characteristics, we used QuintilesIMS, an administrative claims database derived from health plans across the United States, with data for 1,541,873 eligible individuals from 2014-2015. We compared the health care costs, utilization, and medication gap among patients identified through MTM criteria (both broad and narrow, as these are provided as ranges) and our new medication management score markers.

RESULTS: We developed 3 claims-derivable markers: (1) instances when a patient filled a medication with high complexity that could affect adherence, (2) instances where a patient filled a medication defined as costly within a therapeutic category that could affect access, and (3) instances when a patient filled a medication defined as risky that could increase incidence of adverse drug events. In the QuintilesIMS database, individuals with 2 new medication risk markers plus at least 3 conditions and more than $\$ 3,017$ in medication costs when compared with individuals meeting narrow MTM eligibility criteria ( $\geq 8$ medications, $\geq 3$ conditions, and $>\$ 3,017$ medication costs) had increased costs ( $\$ 36,000$ vs $\$ 26,100$ total; $\$ 24,800$ vs 21,400 medical; $\$ 11,300$ vs $\$ 4,800$ pharmacy); acute care utilization ( 0.328 vs 0.256 inpatient admissions and 0.627 vs 0.579 emergency department visits); and 1 or more gaps in medication adherence( $41.5 \%$ vs $34.7 \%)$.

CONCLUSIONS: We identified novel markers of medication use risk that can be determined using insurance claims and can be useful to identify patients for CMM programs and prioritize patients who would benefit from clinical pharmacist intervention. These markers were associated with higher costs, acute care utilization, and gaps in medication use compared with the overall population and within certain subgroups. Providing CMM to these patients may improve health system performance in relevant quality measures. Evaluation of CMM services delivered by a pharmacist using these markers requires further investigation.

Health system pharmacists help achieve the Triple Aim"improving the experience of care, improving the health of populations, and reducing per capita costs of health care"-by optimizing medication use and ensuring the safe and effective delivery of pharmacotherapy to patients. ${ }^{1}$ Improved medication use, achieved through the delivery of comprehensive medication management (CMM) by pharmacists, ${ }^{2,3}$ has been shown to lead to patient satisfaction and trust, reduced medical costs, and improvement in population health. ${ }^{4-6}$ Thus, pharmacists serve an integral role within the interdisciplinary team to deliver high quality and value health care.

The effective integration of pharmacists into the health care workflow to provide CMM for a primary care population can be challenging. There are far more patients who could benefit from pharmacist review and intervention than there are clinical pharmacists available to perform this work. Furthermore, widely accepted standards or automated mechanisms are lacking to identify patients who would most likely benefit from a pharmacist consultation. Tools to prioritize patients for pharmacist review do exist, such as the clinical pharmacy priority (CP2) score, medication user self-evaluation (MUSE) tool, or assessment of risk tool (ART), ${ }^{7-9}$ but many require manual chart review by a pharmacist or data collection via patient survey.

Other criteria, such as medication therapy management (MTM) eligibility criteria from the Centers for Medicare \& Medicaid Services (CMS) Medicare Part D program (ie, $\geq 2-3$ chronic diseases, taking $\geq 3-8$ Part D drugs, annual costs for Part D drugs $\geq \$ 4,044-$ in 2020) ${ }_{1}^{10}$ can be used to identify patients for a pharmacist program but may result in a list of patients that is very large, broad, and not always actionable. Therefore, using these measures to create a priority list for pharmacists often requires a manual, time-consuming process to apply additional criteria such as laboratory data, health care utilization, or other clinical indicators. ${ }^{11-13}$ This process likely varies across providers and settings and may not identify those with the greatest need or patients most likely to benefit from intervention.

The purpose of this study was to describe the process for identifying CMM-related medication-regimen characteristics and develop a tool (referred to internally as the medication management score [MMS]) to identify and prioritize patients in primary care or population-based settings using medical claims data.

\section{Methods}

\section{IDENTIFICATION OF PATIENTS FOR CMM}

In August 2019, we assembled a panel of subject matter experts in outpatient medication use to identify characteristics of patients who would most benefit from CMM. This panel included 5 clinical pharmacists practicing in ambulatory care or adult internal medicine settings within the Johns Hopkins Health System-2 practicing in acute care internal medicine, 1 practicing in ambulatory care internal medicine, 1 practicing in managed care, and 1 serving as a director of clinical services. All 5 completed residency 
training in either ambulatory care, internal medicine, or pharmacotherapy and held at least 1 board certification (pharmacotherapy specialist, ambulatory care pharmacist, and/or geriatrics pharmacist), and 3 had additional degrees (MBA or MPH).

The meeting was conducted by 2 individuals, including 1 investigator and 1 qualitative researcher, and recorded transcripts were reviewed by the project team. The goals of the panel meeting were to solicit participant ideas for definitions of medications at higher risk of adverse drug events (ADEs) and characteristics of ideal candidates for CMM.

We conducted 2 exercises: an unprompted panel discussion and a review of 5 patient cases supplemented with information related to the patient's acute care utilization, recent medical costs, use of opioids, and predicted risk for acute care utilization from the Johns Hopkins Adjusted Clinical Group (ACG) system, which is a risk adjustment tool that uses medical and/or pharmacy claims as inputs and has been validated against health care costs and utilization. ${ }^{14,15}$

\section{DATASET FOR INITIAL EVALUATION OF NOVEL MEDICATION RISK MARKERS}

To assess the prevalence of risk markers developed at the medication level, we used a commercially available National Drug Code (NDC) database (ie, Cerner Multum Lexicon Plus 2019, Cerner Multum), which is a comprehensive database of all prescription drug products available in the US drug market. We considered medications at the NDC or active ingredient levels, such as for measures related to cost and dose form or for measures related to risk of ADEs. We evaluated medication data available as of January 1, 2014, to match our patient-level dataset.

We retrospectively reviewed QuintilesIMS patient-level administrative claims, derived from participating health plans across the United States. Our QuintilesIMS claims data included commercial insurance plans for individuals aged 18-63 years. In addition to demographic and enrollment information, the QuintilesIMS database includes diagnosis codes from International Classification of Diseases, Ninth Revision, Clinical Modification (ICD-9-CM), procedure codes from Current Procedural Terminology, Fourth Edition (CPT-4), or the Healthcare Procedural Coding System (HCPCS); cost information from inpatient, outpatient, and emergency department (ED) settings; and pharmacy claims that included information on medication documented as NDC numbers, quantity, days supply, and cost.

We used 2014 data to construct baseline covariates and claims-based pharmacy markers. We identified 2,034,769 enrollees with continuous medical and pharmacy insurance enrollment in 2014 and 2015 and restricted our sample to
1,541,873 (75.78\%) individuals with complete data who were aged between 18 and 63 years in 2014 .

\section{COMPARISON OF CHARACTERISTICS OF POPULATIONS IDENTIFIED WITH NOVEL MARKERS AND MTM ELIGIBILITY CRITERIA}

We compared patients with novel risk factors to others identified by more traditional criteria, such as MTM eligibility criteria used by CMS, to provide an initial look at populations identified by these measures. We further validated these new measures against health care costs and utilization. ${ }^{16,17}$ We used 2014 QuintilesIMS data to define our outcomes and used MTM eligibility criteria from 2014 supplied by CMS for comparison. ${ }^{18}$ Since MTM eligibility criteria are provided from Medicare as ranges, we compared our markers (alone and in combination with minimum count of chronic conditions and annual pharmacy costs) to 2 sets of criteria (ie, the broadest and the narrowest): (1) "broad" with at least 2 chronic conditions, at least 2 medications, and more than $\$ 3,017$ annual medication costs and (2) "narrow"with at least 3 chronic conditions, at least 8 medications, and at least $\$ 3,017$ annual medication costs.

We evaluated several outcomes in this analysis, including total costs, medical costs, pharmacy costs, count of inpatient admissions, count of ED visits, and medication persistency (ie, absence of clinically relevant gaps in therapy). These outcomes were part of Johns Hopkins ACG system output for these patients.

To identify clinically relevant gaps in therapy, we used the definition included in the ACG system version 12.0. Once the number of days between the end of one prescription and the beginning of the next prescription for the same medication exceeded a critical threshold, the definition of a "gap in therapy" was met. Based on perceived consequences of therapy gaps for different conditions, the ACG system defined a relevant gap as (1) 15 days for chronic conditions that are expected to be particularly sensitive to gaps in therapy (ie, HIV, bipolar, schizophrenia, heart failure, ischemic heart disease, diabetes, depression, and seizure disorder) and (2) 30 days for other chronic conditions not expected to be as sensitive to gaps in therapy.

\section{Results}

OVERVIEW OF 3 NOVEL CLAIMS-BASED RISK MARKERS

Through the results from the expert panel, we identified 3 useful and relevant claims-derivable pharmacy management risk metrics that presently are not routinely applied to electronic data to identify or prioritize patients for CMM by a pharmacist (Supplementary Table 1, available in 


\begin{tabular}{l|l|l|l}
\multicolumn{1}{c|}{ TABLE 1 } & \multicolumn{1}{c}{ Cverview of MMS Markers } & \multicolumn{1}{c}{ Risky medications } \\
\hline Overview & $\begin{array}{l}\text { Medications with complex instruc- } \\
\text { tions for use, including routes of } \\
\text { administration, frequency, or requiring } \\
\text { devices }\end{array}$ & $\begin{array}{l}\text { Medications with inherent risk of } \\
\text { ADEs, including life-threatening com- } \\
\text { plications, or that require therapeutic } \\
\text { drug monitoring for safety }\end{array}$ & $\begin{array}{l}\text { Medications within a therapeutic class } \\
\text { with especially high acquisition costs }\end{array}$ \\
\hline $\begin{array}{l}\text { Definition of } \\
\text { marker }\end{array}$ & $\begin{array}{l}\text { Medications with a dose form/route } \\
\text { score } \geq 3 \text { and frequency score of 3 }\end{array}$ & $\begin{array}{l}\text { Medications identified by published } \\
\text { resources supplemented by expert } \\
\text { opinion }\end{array}$ & $\begin{array}{l}\text { Medications in the top 1\% for AWP } \\
\text { within a therapeutic category }\end{array}$ \\
\hline $\begin{array}{l}\text { Medication level } \\
\text { considered }\end{array}$ & NDC numbers & Active ingredients & $\begin{array}{l}\text { NDC numbers within a therapeutic } \\
\text { class }\end{array}$ \\
\hline $\begin{array}{l}\text { How marker is } \\
\text { derived }\end{array}$ & $\begin{array}{l}\text { Calculated using weights for dose } \\
\text { form, route, and frequency found } \\
\text { in prescription claim using our } \\
\text { Medication Complexity Score, } \\
\text { adapted from MRCl20 }\end{array}$ & $\begin{array}{l}\text { Derived from ISMP,25 AGS Beers } \\
\text { Criteria, 26 and STOPP criteria } \\
\text { additional medication classes via a } \\
\text { panel of pharmacists }\end{array}$ & $\begin{array}{l}\text { Top 1\% AWP threshold calculated } \\
\text { for each therapeutic category from } \\
\text { Multum Drug database }\end{array}$ \\
\hline
\end{tabular}

$A D E s=$ adverse drug events; $A G S=A$ merican Geriatrics Society; $A W P=$ average wholesale price; ISMP=Institute for Safe Medication Practices; $M M S=$ medication management score; $\mathrm{MRCl}=$ Medication Regimen Complexity Index, NDC = National Drug Code; STOPP =Screening Tool of Older Persons' Prescriptions.

online article). These markers indicate when a patient had (1) a medication regimen considered "complex," (2) at least 1 "costly" medication, or (3) at least 1 "risky" medication. For each of these metrics, we developed a standardized, claimsbased pharmacy management risk marker that could be applied to health claims that captured complete information on outpatient medication use over a period of time. An overview of each of these metrics are presented below and visualized in Table 1.

Claims-Based Marker of Medication Complexity. Medications with especially complex instructions are likely to contribute to problems related to medication use due to difficulties with adherence. ${ }^{19}$ These include medications administered multiples times per day, using invasive routes or devices, or conditionally based on patient parameters, such as subcutaneous injection of insulin 3 times daily with meals using a sliding scale. To evaluate medication complexity, we adapted an existing tool, the Medication Regimen Complexity Index (MRCI) for quantifying a patient's regimen complexity. ${ }^{20}$ The MRCI assigns weights to specific dose forms (termed by the developers of that instrument as "component A"; Supplementary Table 3, available in online article), frequencies of doses administered (termed "component B"; Supplementary Table 4, available in online article), and additional instructions such as "as directed" and "with food" or variable doses based on patient circumstances such as pain or blood sugar ("component C") and then provides a complexity score when all components are totaled. Increased medication regimen complexity using the MRCI has been demonstrated to contribute to worsened medication adherence and hospitalizations. ${ }^{21-23}$ However, MRCI relies entirely on access to the patient's electronic health record (EHR), may not include medications prescribed by providers from different health systems, and requires assumptions about continuous use of chronic medications. Thus, an alternative method based on medication claims would be very useful in many settings.

We developed a claims-based medication complexity score (MCS) based on algorithms that assessed the regimen complexity of all concurrent medications filled by outpatient pharmacies using the patient's insurance coverage. Using information from Multum (Supplementary Table 2, available in online article), we derived the dosage form and route of administration from prescription claims for each NDC number and assigned a component A weight following MRCI's algorithm (Supplementary Table 3, available in online article). Among 92,053 eligible unique NDC numbers without missing dosage and route, most had a component $\mathrm{A}$ weight of 1 (higher means more complex): $1=61,210$ (66.5\%), $2=15,906$ (17.3\%), $3=2,373$ (2.6\%), $4=12,178$ (13.2\%), and $5=386(0.4 \%)$. Since a prescription's specific dosing instructions or dosing frequency are not included in medication claims, these were deduced based on quantities dispensed per day (ie, quantity dispensed divided by days of supply).

Subsequently, a component B weight was assigned, but no component $\mathrm{C}$ was assigned (for calculated doses dispensed per day $>24$, we assigned a set component $\mathrm{B}$ weight of 2.) For example, a prescription for 60 tablets of "metformin $500 \mathrm{mg}$ tablets by mouth to be taken twice daily" would include dose form (tablets) and route (by mouth) for MCS component A and quantity dispensed per days supply 
(60 tablets $\div 30$-day supply) for MCS component B. The total score for this medication would be $3(1+2)$. A comparison of weights for our MCS components A and B and MRCI components A and B can be found in Supplementary Tables $\underline{3}$ and 4 (available in online article).

We proposed that a complex medication would involve using an individual prescription with a component A score of at least 3 (dose forms that require increased patient coordination using devices or parenteral administration routes) and a component B score of at least 3 (three or more dosing events per day). We also compared our novel MCS measurement to the original MRCI score, which is discussed in a separate publication. ${ }^{17}$

Risky Medication Marker. We defined high-risk medications as those with narrow therapeutic windows, that require therapeutic drug monitoring, or that are known to interact with other medications or physiology of advanced age. ${ }^{24}$ These include medications from lists such as the Institute for Safe Medication Practices (ISMP) high-alert medications, ${ }^{25}$ American Geriatrics Society (AGS) Beers Criteria, ${ }^{26}$ and Screening Tool of Older Persons' Prescriptions (STOPP) criteria. ${ }^{27}$ They are also called "potentially inappropriate medications." We proposed using a count of unique risky medications, defined by active ingredients or therapeutic classes considered risky by ISMP ${ }^{25}$ AGS Beers Criteria, ${ }^{26}$ and STOPP criteria ${ }^{27}$ supplemented by clinical judgment from a panel of pharmacists (including newer medication classes not specified in these lists, such as direct oral anticoagulants; medication classes related to those specified in these lists, such as benzodiazepines, or medications with Risk Evaluation and Mitigation Strategy programs), as criteria for risky medications.

In addition, we proposed specific subcategories to differentiate these risky medications: anticholinergic medications, anticoagulants or antiplatelet medications, chemotherapy, hypoglycemic medications (including insulin and other medications that can cause hypoglycemia), benzodiazepines and Z-drug hypnotics, opioids, immunosuppressants, and antiretrovirals. Some of the medication classes included in these subcategories have been shown to contribute to risk for ADEs. ${ }^{28}$

To exclude sporadic users of these risky medications (eg, as needed or temporary use of first-generation antihistamines included in the anticholinergic subcategory), we set days supply thresholds for each subcategory: at least 120 days of use per 12 months per unique medication for the anticholinergic subcategory (because these medications are more often used as needed-occasional use is unlikely to have as much of an impact on the patient in terms of adverse reactions or interactions with other medications) and at least 60 days of use per 12 months per unique medication for the other subcategories. These thresholds were identified primarily through group consensus, since no standard definition for persistent medication use exists.

In the Multum database, 628 of 2,778 active ingredients (22.61\%) were considered risky medications. The subcategories included 62 anticholinergic active ingredients (2.23\%), 32 anticoagulant or antiplatelet active ingredients (1.15\%), 231 chemotherapy active ingredients (8.32\%), 32 hypoglycemic active ingredients (1.15\%), 20 benzodiazepines or Z-drug hypnotic active ingredients (0.72\%), 64 immunosuppressant/disease-modifying antirheumatic drug active ingredients (2.30\%), 38 antiretroviral therapy active ingredients (1.37\%), and 108 other risky active ingredients (3.89\%). Opioids (41 active ingredients, 1.48\%) are already considered elsewhere in the ACG system with the high-risk opioids user marker (including persons with 100+ morphine milligram equivalents per day for $90^{+}$consecutive days and persons with $30+$ days of concomitant opioid and benzodiazepine use). ${ }^{29}$

Costly Medication Marker. Medications with high acquisition costs are likely to increase barriers for continuous use by patients, either because of formulary restrictions, requirement to use a specialty pharmacy, or simply because of high out-of-pocket costs. ${ }^{30}$ This effect appears more pronounced in patients with specific chronic conditions, such as depression. ${ }^{31}$ Although patient out-of-pocket costs vary widely depending on insurance coverage, a count of medications with high cost (at least from the health system perspective) within a therapeutic category should be available for use in prioritizing patients for CMM and predicting problems related to medication use. Definitions for "specialty" medications, typically very expensive or with formulary restrictions around which clinical services are often dedicated, are not standardized and thus not currently useful for identifying medications for patient prioritization using claims at this time. We identified medications by NDC number as costly in the top $1 \%$ of average wholesale price (AWP) within a therapeutic category of 10 or more NDC numbers. Among 87,948 eligible NDC numbers in the Multum database, 1,209 (1.37\%) were identified in the top $1 \%$ of their therapeutic categories and flagged as costly.

\section{COMPARISON OF PATIENTS WITH NOVEL MEDICATION MARKERS AND MTM ELIGIBILITY CRITERIA}

Compared with persons meeting broad MTM eligibility criteria ( $\geq 2$ medications, $\geq 2$ conditions, and $>\$ 3,017$ pharmacy costs), persons with any combination of MMS markers plus 

Claims-Based Case-Finding Criteria

\begin{tabular}{|c|c|c|c|c|c|}
\hline \multirow{2}{*}{$\begin{array}{l}\text { Subgroups of patients } \\
\text { Entire cohort }\end{array}$} & \multicolumn{2}{|c|}{$\begin{array}{c}\mathbf{N} \\
\text { (\% of sample) }\end{array}$} & \multirow{2}{*}{$\begin{array}{c}\begin{array}{c}\text { Total costs, } \$ \\
(\text { mean } \pm \text { SD) }\end{array} \\
\$ 4,700 \pm \$ 16,900 \\
\end{array}$} & \multirow{2}{*}{$\begin{array}{c}\begin{array}{c}\text { Medical costs, } \$ \\
(\text { mean } \pm \text { SD) }\end{array} \\
\$ 3,700 \pm \$ 15,200\end{array}$} & \multirow{2}{*}{$\begin{array}{c}\text { Pharmacy costs, } \$ \\
\text { (mean } \pm \text { SD) }\end{array}$} \\
\hline & $1,541,873$ & $(100)$ & & & \\
\hline \multicolumn{6}{|c|}{ Subgroups defined by MTM eligibility criteria ${ }^{b}$} \\
\hline $\begin{array}{l}\text { Broad } \\
\text { ( } \geq 2 \text { conditions, } \geq 2 \text { medications, } \\
>\$ 3,017 \text { annual Part } D \text { cost })\end{array}$ & 224,602 & $(14.6)$ & $\$ 19,800 \pm \$ 36,400$ & $\$ 16,500 \pm \$ 34,100$ & $\$ 3,300 \pm \$ 10,600$ \\
\hline $\begin{array}{l}\text { Narrow } \\
(\geq 3 \text { conditions, } \geq 8 \text { medications, } \\
>\$ 3,017 \text { annual Part } D \text { cost })\end{array}$ & 108,537 & $(7.0)$ & $\$ 26,100 \pm \$ 45,000$ & $\$ 21,400 \pm \$ 42,500$ & $\$ 4,800 \pm \$ 12,300$ \\
\hline \multicolumn{6}{|c|}{ Subgroups defined by novel MMS markers ${ }^{\complement}$ plus broad criteria ( $\geq 2$ conditions, $>\$ 3,017$ annual medications cost) } \\
\hline 1+ complex medication & 9008 & $(0.1)$ & $\$ 28,400 \pm \$ 51,600$ & $\$ 22,000 \pm \$ 47,500$ & $\$ 6,400 \pm \$ 16,900$ \\
\hline $1+$ risky medication & 82,743 & $(5.4)$ & $\$ 27,500 \pm \$ 47,100$ & $\$ 20,900 \pm \$ 43,600$ & $\$ 6,600 \pm \$ 15,800$ \\
\hline $1+$ costly medication & 14,609 & $(0.9)$ & $\$ 25,700 \pm \$ 44,700$ & $\$ 17,600 \pm \$ 37,600$ & $\$ 8,100 \pm \$ 21,800$ \\
\hline Any 1 MMS marker & 84,124 & $(5.5)$ & $\$ 25,100 \pm \$ 44,000$ & $\$ 18,90019,600 \pm \$ 39,60041,300$ & $\$ 5,500 \pm \$ 12,900$ \\
\hline Any 2 MMS markers & 10,389 & $(0.7)$ & $\$ 34,600 \pm \$ 54,800$ & $\$ 23,500 \pm \$ 47,700$ & $\$ 11,600 \pm \$ 24,700$ \\
\hline All 3 MMS markers & 486 & $(0.0)$ & $\$ 53,100 \pm \$ 81,200$ & $\$ 31,700 \pm \$ 70,800$ & $\$ 21,500 \pm \$ 36,500$ \\
\hline \multicolumn{6}{|c|}{ Subgroups defined by novel MMS markers ${ }^{c}$ plus narrow criteria ( $\geq 3$ conditions, $>\$ 3,017$ annual medications cost) } \\
\hline 1+ complex medication & 7,799 & $(0.5)$ & $\$ 23,700 \pm \$ 50,100$ & $\$ 23,700 \pm \$ 50,100$ & $\$ 6,700 \pm \$ 15,800$ \\
\hline $1+$ risky medication & 71,605 & $(4.6)$ & $\$ 29,000 \pm \$ 49,200$ & $\$ 22,200 \pm \$ 45,800$ & $\$ 6,800 \pm \$ 15,900$ \\
\hline $1+$ costly medication & 12,228 & $(0.8)$ & $\$ 27,600 \pm \$ 47,400$ & $\$ 19,000 \pm \$ 40,100$ & $\$ 8,600 \pm \$ 22,800$ \\
\hline Any 1 MMS marker & 71,535 & $(4.6)$ & $\$ 26,600 \pm \$ 46,200$ & $\$ 20,900 \pm \$ 43,600$ & $\$ 5,600 \pm \$ 13,000$ \\
\hline Any 2 MMS markers & 9,375 & $(0.6)$ & $\$ 36,000 \pm \$ 56,400$ & $\$ 24,800 \pm \$ 49,700$ & $\$ 11,300 \pm \$ 24,900$ \\
\hline All 3 MMS markers & 449 & $(0.0)$ & $\$ 55,400 \pm \$ 83,600$ & $\$ 33,400 \pm \$ 73,300$ & $\$ 21,900 \pm \$ 37,200$ \\
\hline \multicolumn{6}{|c|}{$\begin{array}{l}{ }^{\circ} \text { These eligibility criteria are defined by CMS for the Medicare program. The cost threshold is defined each year and involves the likely costs of covered Part } D \\
\text { drugs ( } \$ 3,017 \text { for } 2014) .^{18} \text { The chronic conditions assessed can either include any chronic diseases or specific chronic diseases as defined by the Part } D \text { plan } \\
\text { (eg, diabetes, dyslipidemia, and end-stage renal disease). This analysis used any chronic condition. }\end{array}$} \\
\hline \multicolumn{6}{|c|}{ 'This represents the novel high-risk markers developed by this project and described in this study. } \\
\hline \multicolumn{6}{|c|}{$C M S=$ Centers for Medicare \& Medicaid Services; MMS=medication management score; $M T M=$ medication therapy management.} \\
\hline
\end{tabular}

at least 2 chronic conditions and more than \$3,017 pharmacy costs had higher total costs, medical costs, pharmacy costs, utilization, and adherence gaps (Table 2). Compared with persons meeting narrow MTM eligibility criteria $(\geq 8$ medications, $\geq 3$ conditions, and $>\$ 3,017$ pharmacy costs), persons with 2 MMS markers plus at least 3 chronic conditions and more than $\$ 3,017$ pharmacy costs had higher total costs ( $\$ 36,000$ vs $\$ 26,100)$, medical costs ( $\$ 24,800$ vs 21,400$)$, pharmacy costs ( $\$ 11,300$ vs $\$ 4,800$ utilization ( 0.328 vs 0.256 inpatient admissions and 0.627 vs $0.579 \mathrm{ED}$ visits), and 1 or more gaps in medication use (41.4\% vs $34.7 \%$; Table 3 ).

Comparing the 1,541,873 eligible individuals, persons with any complex, risky, or costly medication had increased total costs vs persons meeting broad MTM eligibility ( $\$ 13,700$, $\$ 14,700$, and $\$ 15,200$ vs $\$ 4,700)$; medical costs $(\$ 9,700$, $\$ 9,700$, and $\$ 9,300$ vs $\$ 3,700)$; pharmacy costs $(\$ 4,000$, $\$ 5,000$, and $\$ 5,900$ vs $\$ 1,000)$; utilization $(0.130,0.104$, and 0.091 vs 0.032 inpatient admissions; $0.415,0.276$, and 0.251 vs $0.165 \mathrm{ED}$ visits); and 1 or more gaps in therapy (26.9\%, $27.2 \%$, and $22.5 \%$ vs $9.3 \%$ of subgroup; Supplementary Tables 5 and 6 , available in online article).

The cost outcomes for similar subgroups are summarized in Table 2, and utilization-related outcomes and adherence gaps for similar subgroups are summarized in Table 3. All data are available in the Supplementary Materials (available in online article). 
Comparison of Utilization and Adherence Gaps (Per Annum) Within Subgroups Identified Using Current and Novel Claims-Based Case-Finding Criteria

\begin{tabular}{|c|c|c|c|c|c|}
\hline \multirow{2}{*}{$\begin{array}{l}\text { Subgroups of patients } \\
\text { Entire cohort }\end{array}$} & $\begin{array}{c}\mathrm{N} \\
\text { (\% of sample) }\end{array}$ & \multirow{2}{*}{ 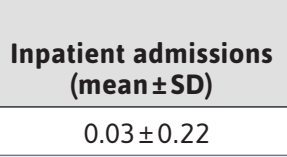 } & \multirow{2}{*}{$\begin{array}{c}\begin{array}{c}\text { ED visits } \\
\text { (mean } \pm \text { SD) }\end{array} \\
0.17 \pm 0.56\end{array}$} & \multicolumn{2}{|c|}{$\begin{array}{l}\text { Patients with } 1+ \\
\text { Rx gaps }^{\mathrm{b}} \mathrm{n} \text {, rounded } \\
\text { (\% of subgroup) }\end{array}$} \\
\hline & $1,541,873(100)$ & & & 142,800 & (9.3) \\
\hline \multicolumn{6}{|l|}{ MTM eligibility criteriac } \\
\hline $\begin{array}{l}\geq 2 \text { conditions, } \geq 2 \text { medications, }>\$ 3,017 \\
\text { annual medication cost }\end{array}$ & $224,602(14.6)$ & $0.19 \pm 0.52$ & $0.48 \pm 1.05$ & 55,200 & $(24.6)$ \\
\hline $\begin{array}{l}\geq 3 \text { conditions, } \geq 8 \text { medications, }>\$ 3,017 \\
\text { annual medication cost }\end{array}$ & 108,537 & $0.26 \pm 0.63$ & $0.58 \pm 1.24$ & 37,700 & $(34.7)$ \\
\hline \multicolumn{6}{|c|}{ Subgroups defined by novel MMS markers ${ }^{d}$ plus broad criteria ( $\geq 2$ conditions, $>\$ 3,017$ annual medication cost) } \\
\hline 1+ complex medication & 9,008 & $0.32 \pm 0.74$ & $0.75 \pm 1.45$ & 3,600 & (39.9) \\
\hline $1+$ risky medication & 82,743 & $0.24 \pm 0.62$ & $0.51 \pm 1.17$ & 27,400 & (33.1) \\
\hline $1+$ costly medication & 14,609 & $0.20 \pm 0.55$ & $0.43 \pm 1.03$ & 4,200 & $(28.6)$ \\
\hline Any 1 MMS marker & 84,124 & $0.22 \pm 0.58$ & $0.49 \pm 1.11$ & 26,200 & $(31.2)$ \\
\hline Any 2 MMS markers & 10,389 & $0.30 \pm 0.72$ & $0.60 \pm 1.39$ & 4,100 & (39.3) \\
\hline All 3 MMS markers & $(0.0)$ & $0.47 \pm 0.97$ & $0.69 \pm 1.65$ & 200 & $(47.5)$ \\
\hline \multicolumn{6}{|c|}{ Subgroups defined by novel MMS markers ${ }^{\mathrm{d}}$ plus narrow criteria ( $\geq 3$ conditions, $>\$ 3,017$ annual medication cost) } \\
\hline 1+ complex medication & 7,799 & $0.35 \pm 0.78$ & $0.77 \pm 1.50$ & 3,300 & $(42.6)$ \\
\hline $1+$ risky medication & 71,605 & $0.27 \pm 0.65$ & $0.53 \pm 1.21$ & 25,600 & $(35.7)$ \\
\hline $1+$ costly medication & 12,228 & $0.22 \pm 0.59$ & $0.45 \pm 1.07$ & 3,900 & $(31.5)$ \\
\hline Any 1 MMS marker & 71,535 & $0.25 \pm 0.61$ & $0.51 \pm 1.15$ & 24,300 & $(34.0)$ \\
\hline Any 2 MMS markers & 9,375 & $0.33 \pm 0.75$ & $0.63 \pm 1.43$ & 3,900 & $(41.4)$ \\
\hline All 3 MMS markers & $(0.0)$ & $0.49 \pm 1.00$ & $0.72 \pm 1.70$ & 200 & $(49.7)$ \\
\hline
\end{tabular}

a Data source: National sample of commercially insured continuously enrolled population aged 18-64 years for calendar year 2014.

${ }^{b}$ Gaps in adherence assessed for a subset of 11 therapeutic categories using ACG software version 12.0. A gap was defined as any interval between the end of the days supply of the first prescription and the onset of a new prescription for the same medication greater than the defined grace period.

'These eligibility criteria are defined by CMS for the Medicare program. The cost threshold is defined each year and involves the likely costs of covered Part D drugs $\left(\$ 3,017\right.$ for 2014). ${ }^{18}$ The chronic conditions assessed can either include any chronic diseases or specific chronic diseases as defined by the Part D plan (eg, diabetes, dyslipidemia, and end-stage renal disease). This analysis used any chronic condition.

${ }^{d}$ This represents the novel high-risk markers developed by this project and described in this study.

ACG = Johns Hopkins Adjusted Clinical Group system; CMS=Centers for Medicare \& Medicaid Services; $M M S=$ medication management score; $M T M=m e d i c a t i o n$ therapy management.

\section{Discussion}

Identifying and prioritizing patients for clinical pharmacy services in primary care is challenging, especially when using manual chart review, and we believe efficiencies can be gained using claims data. The key contribution of our project is the development of 3 novel claims-based markers for potential use in support of primary care/ population-based CMM programs. This study provides an overview of the rationale, components, specifications, and properties of the markers. As noted, our marker development process included qualitative input from subject matter experts-a panel of practicing adult primary care clinical pharmacists at our academic medical center. In addition, we conducted an in-depth empirical exploration using medical and pharmacy claims from insured populations to assess medical costs and utilization in populations identified using these markers.

Our newly developed markers provided improved patient identification and prioritization compared with MTM eligibility criteria in our sample population. Compared with the MTM eligible subgroups and the overall population, persons meeting criteria similar to the MTM eligibility criteria (both broad and narrow, including a minimum number of chronic 
conditions and annual pharmacy costs) and who take medications defined by our MMS markers have increased total costs, medical costs, pharmacy costs, inpatient admissions, ED visits, and gaps in medication adherence.

Although MTM eligibility criteria were not designed to identify a subpopulation of patients for pharmacist review, we propose that our MMS markers can be used to identify patients for CMM. Patients who have 2 or more MMS markers are more likely to have higher costs and health care utilization than patients meeting MTM eligibility criteria. We found similar proportions of patients with gaps in adherence when comparing patients who have MMS markers and who meet MTM eligibility criteria. We also found some MMS subgroups without a minimum medication regimen size that have fewer adherence gaps than narrow MTM eligibility criteria.

This project is part of a larger effort that will eventually operationalize these and similar markers as part of ACG risk adjustment software. We plan to use the MMS markers in combination with other data from the ACG system, such as patient demographics, clusters of chronic conditions, adherence measures, or other clinical targets, and anticipate this to improve the specificity of these tools for identifying patients within a claims database and prioritizing work for pharmacists providing CMM. We also intend to explore various other thresholds for our markers, such as top $5 \%$ cost or other combinations of complexity scores as we continue to develop these tools.

Other prioritization tools in published literature, such as the CP2 score and MUSE, ${ }^{7,8}$ consider the number of a patient's medications but do not consider specific medication-level characteristics. For example, a patient would receive a higher $\mathrm{CP} 2$ score based on the number of "active items on medication list" but without differentiation of these medications or medication classes. In addition, many of these scores do not consider whether prescribed medications are filled, generating prescription claims. Therefore, our new MMS markers can be viewed as an improvement of these existing tools.

Our MMS pharmacy management risk markers can be applied on a routine basis using available claims or other similar databases, in combination with other patient characteristics, to automatically identify and prioritize patients for CMM. This automation allows pharmacists on interdisciplinary care teams to focus their time providing care directly to the right patients-those who are most likely to have problems related to medication use that leads to increased cost and utilization.

\section{LIMITATIONS}

This study has some limitations to consider. Our subject matter expert panel consisted of 5 clinical pharmacists practicing within our multicenter academic health system, a group that did not include pharmacists such as consultant pharmacists in the community who also deliver CMM in the primary care or population health setting. We opted to focus on subject matter experts within our health system and did not use qualitative research methods due to limited resources. In addition, we did not include physicians or other clinicians who interact with medications on this panel, although other providers were included on our project team.

Because our suggested MCS component B required prescription claims for its calculation, the prevalence of MCS component B and overall MCS scores cannot be assessed with only medication data from Multum as was the case with component A. Although our score for medication complexity (MCS) performs similarly to $\mathrm{MRCI},{ }^{17}$ comparison of our MCS against MRCI and validation against patient outcomes is needed before it is used in practice.

Our marker for costly medications relied on AWP, an imperfect measure of cost. Because of factors such as insurance coverage, formulary status, and deductibles, using AWP to accurately estimate a patient's out-of-pocket costs was challenging. This marker may be most helpful to identify high-cost medication use where less expensive alternatives exist within the same medication class (eg, high-cost branded product vs a lower-cost generic product). Unlike the other markers described here, the costly medications marker using AWP likely had less applicability to health systems with closed formularies (where medications are selected using negotiated prices or rebates, with less flexibility for alternatives considered by our marker) or outside the United States, with differing levels of government subsidization or negotiation of medication costs.

The risks and severity of ADEs or other negative patient outcomes for our proposed risky medication subcategories are admittedly different. For example, although ADEs from combinations of anticholinergic medications and their sequelae are potentially harmful for patients, especially if older or frail, ${ }^{32}$ ADEs from anticoagulants can be directly fatal. ${ }^{33}$ The MedWise Risk Score considers medication-level characteristics, ${ }^{12,13}$ including a scheme for differentiating risky medications into domains, which is an improvement over an admittedly heterogenous group of risky medications identified by ISMP or other organizations. We differentiated our high-risk medications into subcategories; empirically weighting these subcategories is an area for future research. In addition, we did not consider interactions between medications and between medications 
and patient characteristics (eg, renal impairment), and this is also an area for future research.

Our commercial insurance claims database did not include patients aged less than 18 years and aged 65 years or older, with fee-for-service Medicare or Medicaid data. These populations can have different demographics, medication use, and health care utilization when compared with commercially insured adult patients. ${ }^{34,35}$ Moreover, the MTM eligibility criteria used for comparison was specifically intended for use in the Medicare population. The impact of our proposed markers on other populations, including patients with Medicare and Medicaid, is an area for future research.

Medical claims data using ICD9-CM diagnostic codes are not very reliable for identifying the incidence of ADEs, ${ }^{36,37}$ so we opted not to include this as an outcome.

We elected to use medication persistency as our quality metric related to medication use. This decision was made pragmatically, since it is already assessed in the ACG software to which our work is related. Future efforts will be made to integrate measures of medication adherence, such as the proportion of days covered within an interval that a patient has drug supply according to their fill records.

Beyond the characteristics that we have identified (eg, complexity, risk, and cost), there are several patient characteristics highlighted by our expert panel that are not commonly available either in patient EHRs or claims data, such as social determinants of health and health literacy. These factors likely affect medication use and adherence and should be areas for future development. ${ }^{38}$

Finally, these markers and future tools that we aim to develop are intended for health systems or pharmacy departments with access to claims data and analytical capability.

\section{Conclusions}

Our team identified 3 novel markers of potential pharmacy use risk that can be determined for each member of a population using existing electronic data. Since the process is automated using computing power, it makes operations more efficient by obviating the need for manual chart review. We used the markers to identify patients whose medical costs and medication nonadherence levels were higher than in patients selected using standard criteria. These patients may comprise a group that is more likely to benefit from a pharmacist's CMM activities. Empirical assessment of this hypothesis, including in patients aged 65 years and older, is underway.

\section{DISCLOSURES}

No outside funding supported this study. All authors are Johns Hopkins employees. The Johns Hopkins University receives royalties for nonacademic use of software based on the Johns Hopkins Adjusted Clinical Group (ACG) methodology. Chang, Kitchen, Weiner, and Kharrazi receive a portion of their salary support from this revenue. The authors have no conflicts of interests relevant to this study.

\section{REFERENCES}

1. Berwick DM, Nolan TW, Whittington J. The triple aim: care, health, and cost. Health Aff (Millwood). 2008;27(3):759-69.

2. The Patient Centered Primary Care Collaborative. The patient-centered medical home: integrating comprehensive medication management to optimize patient outcomes. 2nd ed. Updated 2012. Accessed July 10, 2020. http://www. pcpcc.org/sites/default/files/media/ medmanagement.pdf

3. Pestka DL, Frail CK, Sorge LA, Funk KA, Roth McClurg MT, Sorensen TD. The practice management components needed to support comprehensive medication management in primary care clinics. J Am Coll Clin Pharm. 2020;3(2):438-47.
4. Pinto SL, Kumar J, Partha G, Bechtol RA. Pharmacist-provided medication therapy management (MTM) program impacts outcomes for employees with diabetes. Popul Health Manag. 2014;17(1):21-27.

5. Jones LK, Greskovic G, Grassi DM, et al. Medication therapy disease management: Geisinger's approach to population health management. Am J Health Syst Pharm. 2017;74(18):1422-35.

6. Altavela JL, Dorward KM, Sorrento TA Diehl KM, Wyman CA. Population health management: an independent physician organization approach. Am J Health Syst Pharm. 2017;74(18):1477-85.

7. Vande Griend JP, Saseen JJ, Bislip D, Emsermann C, Conry C, Pace WD. Prioritization of patients for comprehensive medication review by a clinical pharmacist in family medicine. J Am Board Fam Med. 2015;28(3):418-24.

8. Doucette WR, Chang EH, Pendergast JF, Wright KB, Chrischilles EA, Farris KB. Development and initial assessment of the medication user self-evaluation (MUSE) tool. Clin Ther. 2013;35(3):344-50.

9. Falconer N, Liow D, Zeng I, Parsotam N, Seddon M, Nand S. Validation of the assessment of risk tool: patient prioritisation technology for clinical pharmacist interventions. Eur J Hosp Pharm. 2017;24(6):320-26.

10. Chavez-Valdez AL. CY 2020 medication therapy management program guidance and submission instructions. April 5, 2019. Accessed July 10, 2020. https://www. cms.gov/Medicare/Prescription-DrugCoverage/PrescriptionDrugCovContra/ Downloads/Memo-Contract-Year-2020Medication-Therapy-Management-MTMProgram-Submission-v-041019-.pdf

11. Lee JS, Yang J, Stockl KM, Lew H, Solow BK. Evaluation of eligibility criteria used to identify patients for medication therapy management services: a retrospective cohort study in a Medicare Advantage Part D population. J Manag Care Spec Pharm. 2016;22(1):22-30. doi: 10.18553/jmcp.2016.22.1.22 
12. Cicali B, Michaud V, Knowlton $\mathrm{CH}$, Turgeon J. Application of a novel medication-related risk stratification strategy to a self-funded employer population. Benefits Q. 2018;34(2):49-55.

13. Bankes DL, Jin H, Finnel S, et al. Association of a novel medication risk score with adverse drug events and other pertinent outcomes among participants of the programs of all-inclusive care for the elderly. Pharmacy (Basel). 2020;8(2):87.

14. Weiner JP, Starfield BH, Steinwachs DM, Mumford LM. Development and application of a population-oriented measure of ambulatory care case-mix. Med Care. 1999;29(5):452-72.

15. Lemke KW, Weiner JP, Clark JM. Development and validation of a model for predicting inpatient hospitalization. Med Care. 2012;50(2):131-39.

16. Ma X, Jung C, Chang HY, Richards TM, Kharrazi H. Assessing the populationlevel correlation of medication regimen complexity and adherence indices using electronic health records and insurance claims. J Manag Care Spec Pharm. 2020;26(7):860-71. doi: 10.18553/ jmcp.2020.26.7.860

17. Kharrazi H, Ma X, Chang HY, Richards TM, Jung C. Comparing the predictive effects of patient Medication adherence indices in electronic health record and claims-based risk stratification models. Popul Health Manag. Online ahead of print February 5, 2021. doi: 10.1089/ pop.2020.0306

18. Centers for Medicare \& Medicaid Services. 2014 Medicare Part D medication therapy management (MTM) programs. Fact sheet. August 21, 2014. Accessed August 26, 2020. https://www. cms.gov/medicare/prescription-drugcoverage/prescriptiondrugcovcontra/ downloads/cy2014-mtm-fact-sheet.pdf

19. Conn VS, Taylor SG, Kelley S. Medication regimen complexity and adherence among older adults. Image J Nurs Sch. 1991;23(4):231-35.

20. George J, Phun Y, Bailey MJ, Kong DCM, Stewart K. Development and validation of the Medication Regimen Complexity Index. Ann Pharmacother. 2004;38(9):1369-1376.
21. Willson MN, Greer CL, Weeks DL. Medication regimen complexity and hospital readmission for an adverse drug event. Ann Pharmacother. 2014;48(1):26-32.

22. Bazargan M, Smith J, Yazdanshenas H, Movassaghi M, Martins D, Orum G. Nonadherence to medication regimens among older African-American adults. BMC Geriatr. 2017;17(1):163.

23. Alves-Conceição V, Rocha KSS, Silva FVN, Silva ROS, Silva DTd, de Lyra DP Jr. Medication regimen complexity measured by MRCI: a systematic review to identify health outcomes. Ann Pharmacother. 2018;52(11):1117-34.

24. Weddle SC, Rowe AS, Jeter JW, Renwick RC, Chamberlin SM, Franks AS. Assessment of clinical pharmacy interventions to reduce outpatient use of high-risk medications in the elderly. J Manag Care Spec Pharm. 2017;23(5): 520-24. doi: 10.18553/jmcp.2017.23.5.520

25. Cohen MR, Smetzer JL, Westphal JE, Comden SC, Horn DM. Risk models to improve safety of dispensing highalert medications in community pharmacies. J Am Pharm Assoc (2003). 2012;52(5):584-602.

26. 2019 American Geriatrics Society Beers Criteria Update Expert Panel. American Geriatrics Society 2019 updated AGS Beers Criteria for potentially inappropriate medication use in older adults. J Am Geriatr Soc. 2019;67(4):674-94.

27. O'Mahony D, O'Sullivan D, Byrne S, O'Connor MN, Ryan C, Gallagher P. STOPP/START criteria for potentially inappropriate prescribing in older people: version 2. Age Ageing. 2015;44(2):213-18.

28. Digmann R, Thomas A, Peppercorn S, et al. Use of Medicare administrative claims to identify a population at high risk for adverse drug events and hospital use for quality improvement. J Manag Care Spec Pharm. 2019;25(3):402-10. doi: 10.18553/jmcp.2019.25.3.402

29. Chang HY, Kharrazi H, Bodycombe D, Weiner JP, Alexander GC. Healthcare costs and utilization associated with high-risk prescription opioid use: a retrospective cohort study. BMC Med. 2018;16(1):69.
30. Morgan SG, Lee A. Cost-related non-adherence to prescribed medicines among older adults: a cross-sectional analysis of a survey in 11 developed countries. BMJ Open. 2017;7(1):e014287.

31. Briesacher B, Gurwitz J, Soumerai S. Patients at-risk for cost-related medication nonadherence: a review of the literature. J Gen Intern Med. 2007;22(6):864-71.

32. Ness J, Hoth A, Barnett MJ, Shorr RI, Kaboli PJ. Anticholinergic medications in community-dwelling older veterans: prevalence of anticholinergic symptoms, symptom burden, and adverse drug events. Am J Geriatr Pharmacother. 2006;4(1):42-51.

33. Piazza G, Nguyen TN, Cios D, et al. Anticoagulation-associated adverse drug events. Am J Med. 2011;124(12):1136-42.

34. Allen LA, Smoyer Tomic KE, Wilson KL, Smith DM, Agodoa I. The inpatient experience and predictors of length of stay for patients hospitalized with systolic heart failure: comparison by commercial, Medicaid, and Medicare payer type. J Med Econ. 2012;16(1):43-54.

35. Hinman A, Bozic KJ. Impact of payer type on resource utilization, outcomes and access to care in total hip arthroplasty. J Arthroplasty. 2008;23

(6 Suppl 1):9-14.

36. Hougland P, Xu W, Pickard S, Masheter C, Williams SD. Performance of International Classification of Diseases, 9th Revision, Clinical Modification codes as an adverse drug event surveillance system. Med Care. 2006;44(7):629-36.

37. Kuklik N, Stausberg J, Jöckel K. Adverse drug events in German hospital routine data: a validation of International Classification of Diseases, 10th Revision (ICD-10) diagnostic codes. PloS One. 2017;12(11):e0187510.

38. Gellad WF, Grenard JL, Marcum ZA. A systematic review of barriers to medication adherence in the elderly: looking beyond cost and regimen complexity. Am J Geriatr Pharmacother. 2011;9(1):11-23. 